\title{
INVESTIGAÇÃO SOBRE O USO DA LÓGICA DE PROGRAMAÇÃ̃O NO ENSINO MÉDIO BRASILEIRO: UMA REVISÃO SISTEMÁTICA
}

\author{
Natália Bernardo Nunes, Aline Silva de Bona e Anelise Lemke Kologeski \\ Instituto Federal de Educação, Ciência e Tecnologia do Rio Grande do Sul - Canpus Osório \\ Rua Santos Dumont, 2127, Osório/RS, Brasil, 95520-000
}

\begin{abstract}
RESUMO
O Brasil é o $7^{\circ}$ produtor de TICs no mundo, enquanto decresce ano a ano na educação. Por isso, uma investigação é realizada, analisando a eficiência da lógica de programação no Ensino Médio, a fim de buscar alternativas para inovar a educação, bem como uma forma de aplicar os conceitos essenciais da Computação no cotidiano dos estudantes. Uma revisão sistemática foi desenvolvida, em conjunto com uma pesquisa de campo, investigando a perspectiva dos educandos. Foram encontrados 41 artigos voltados aos critérios propostos, e uma grande diferença entre as regiões do Brasil e os anos de publicação, enquanto que nas entrevistas, com a adesão de 366 estudantes, observou-se que a temática abordada pode sim influenciar nos índices educacionais, mostrando-se de grande importância na vida dos participantes.
\end{abstract}

\section{PALAVRAS-CHAVE}

Pensamento Computacional, Computação, Educação, Raciocínio Lógico, Tecnologia

\section{INTRODUÇÃO}

A tecnologia, ao longo dos anos, está se tornando um tema de grande interesse e familiaridade, principalmente àqueles jovens que fazem parte da geração $\mathrm{Z}$, também conhecidos como nativos digitais, ou seja, só conhecem a linguagem digital e passam grande parte de sua vida online, sem distinguir sua identidade entre o campo online e offline (Palfrey e Gasser, 2011). Quanto à tecnologia no mercado, pode ser observado que o Brasil é o $7^{\circ}$ maior produtor de Tecnologia da Informação e Comunicação do mundo, segundo dados da Brasscom (Brasscom, 2019).

No entanto, há uma grande controvérsia perante a este grande avanço. O Índice de Desenvolvimento da Educação Básica (IDEB, 2020) apresenta baixos índices educacionais no Brasil, principalmente com estudantes do Ensino Médio, calculados por meio de uma prova bianual que mensura com notas de 0 a $10 \mathrm{o}$ desempenho dos estudantes brasileiros do ensino básico, em competências relacionadas os conhecimentos básicos necessários em Língua Portuguesa e Matemática. Segundo dados encontrados na plataforma do IDEB, nas três últimas edições do exame, o resultado atingido por educandos do Ensino Médio foi abaixo do índice esperado pelo órgão. Além deste fator, é relevante considerar o Ranking Mundial de Competitividade publicado pelo Institute for Management Development (IMD) em 2020, onde o Brasil se encontra em último lugar em educação (IMD, 2020) enfatizando assim que a qualidade do ensino nas escolas ainda carece de melhorias.

A presidente do Instituto Ayrton Senna, Viviane Senna, em entrevista ao canal BBC News Brasil, declara para Costas (2015) que, se um cirurgião, um operador de bolsas ou até mesmo um piloto de avião do século XIX fossem trazidos à atualidade, eles não saberiam o que fazer em seus ambientes de trabalho, devido às grandes mudanças que ocorreram ao longo dos séculos. Por outro lado, um professor colocado na mesma situação saberia exatamente o que fazer, já que encontraria o mesmo formato de escola, com alunos distribuídos em classes, enfileirados, e uma lousa para a escrita. Posteriormente, Senna reafirma para Idoeta (2019) em uma entrevista, no mesmo veículo de comunicação, que o Brasil ainda sofre com déficits educacionais do século XIX, além de enfrentar necessidade em todos os demais setores, para atender às demandas do século XXI. Com estas afirmações, é possível observar que existe uma relação com a 
inadequação da educação escolar às exigências do século XXI e aos poucos indícios de práticas inovadoras voltadas para o Ensino Médio, justamente em um momento onde os estudantes estão se preparando para ingressar no mercado de trabalho e/ou no ensino superior, e que exige mudanças muito rapidamente devido aos avanços tecnológicos, demandando um conhecimento mínimo para o uso dos mais diversos recursos da Tecnologia da Informação e Comunicação (TDIC) que conhecemos e utilizamos. Neste contexto, de acordo com (Schuhmacher et. al., 2016), "para a escola se estabelece o desafio e a incumbência de formar e preparar o novo cidadão para enfrentar os desafios que ainda estão por vir e que a sociedade lhe exige, sem saber com certeza quais são, e muito menos como ensiná-las".

Assim, evidencia-se que métodos inovadores na Educação precisam emergir para que as demandas da sociedade sejam devidamente atendidas. Um exemplo de atitude que vai ao encontro desta necessidade pode ser observada nos países que estão em colocações acima do Brasil no Ranking Mundial de Educação do Programa Internacional de Avaliação de Estudantes (Lyceum, 2019). Esses países estão passando a implementar a computação no currículo escolar, com metodologias variadas de acordo com o país (Heintz et. al., 2016). Esse fato sugere uma reflexão, questionando se esta também seria uma solução eficiente para o Brasil. Para (Schuhmacher et. al., 2016), a inserção de conceitos de Computação na educação básica está sendo estimulada enquanto parte estruturante do currículo, através da introdução do ensino de programação nos currículos, por meio de estratégias que privilegiam a perspectiva do desenvolvimento de atividades que explorem a criação de algoritmos de desenvolvimento de produtos, seguindo para a aprendizagem de linguagem de programação e abstração de conceitos computacionais apoiando o desenvolvimento do Pensamento Computacional. Contudo, de acordo com (Gomes e Melo, 2013) o processo de ensino-aprendizagem de conceitos fundamentais de computação - conhecimentos de lógica de programação, estruturação de algoritmos e a abstração necessária à resolução de problemas computacionais - é potencialmente desafiador, apresentando dificuldades em vários aspectos, quer seja sob a perspectiva do professor ou do aluno, gerando discussões acerca de métodos, técnicas e ferramentas, bem como aspectos cognitivos e pedagógicos envolvidos.

Ainda considerando dados do Ranking Mundial de Competitividade da IMD, observa-se que Singapura é o país que lidera a mesma classificação educacional na qual o Brasil se encontra em último lugar. Tornando este líder da lista como referência, observa-se que existe uma movimentação envolvendo a inclusão digital desde a formação docente. Silva (2011) aponta em sua dissertação que o Instituto Nacional de Educação de Singapura possui em sua ementa do curso de pedagogia a disciplina de TICs como Ferramenta de Aprendizagem Significativa I e II, preparando especialistas para exercerem sua profissão com o uso de novas tecnologias, atendendo assim de forma mais adequada às demandas do século XXI.

Considerando estes fatores como critério relevante para a análise da inclusão da lógica programação no sistema educacional, a implementação da mesma torna-se ainda mais importante no Ensino Médio, visto que uma revisão sistemática realizada em 2018, que contou com 20 artigos publicados em eventos de referência na área, aponta que, enquanto 65\% das atividades de computação desplugada publicadas eram voltadas ao Ensino Fundamental, apenas 35\% delas abrangiam o público do Ensino Médio (Rodrigues et. al., 2018). Esta afirmação reforça que há uma carência de atividades computacionais para este nível de ensino, justamente em um momento onde a maioria dos estudantes busca uma qualificação tecnológica mínima para a competitiva busca por uma vaga no mercado de trabalho.

Um outro ponto que reforça essa ideia são os dados de um estudo que também utiliza o método de Revisão Sistemática de Literatura em busca de trabalhos envolvendo o Pensamento Computacional por meio de objetos de aprendizagem, no qual foram selecionados 12 principais artigos, e apenas 3 deles eram direcionados ao Ensino Médio (Carvalho et. al., 2017). Ademais, torna-se de interesse da faixa etária um modelo pedagógico de inclusão digital, tendo em vista que a maioria dos estudantes estão ingressando ou se preparando para ingressar no mercado de trabalho, que vem se modernizando de forma cada vez mais rápida ao longo dos anos (Brasscom, 2019). Contudo, de acordo com (Sales, 2016) pensar a escola como tendo a obrigação de somente preparar para o mercado de trabalho leva, impreterivelmente, a repensar a qualidade e função da escola, para que esta não se reduza a uma ótica exclusivamente mercantil. Ainda, conforme (Sales, 2016), a educação não pode ser entendida apenas como um instrumento de mobilidade social, mas como uma possibilidade de enriquecimento pessoal. E, por isso, a oferta de atividades que permitam o desenvolvimento de múltiplas habilidades se torna complementar ao ensino tradicional em sala de aula e, ao mesmo tempo, essencial para um aprendizado rico e significativo aos estudantes que participam de iniciativas como as que 
serão investigadas neste trabalho, muitas vezes os motivando a seguir carreira pela área tecnológica, quando envolvem assuntos como lógica de programação, raciocínio lógico e pensamento computacional.

Assim, com base nos trabalhos de (Rodrigues et. al., 2018) e (Carvalho et. al., 2017), reforça-se a ideia de que o Pensamento Computacional e a lógica de programação não estão associados apenas com a Computação, mas sim com as atividades mais básicas do cotidiano, e se o educando possui conhecimento deste assunto, ele desenvolve mais autonomia e capacidade para lidar com a resolução de problemas, aplicando devidamente os conhecimentos relacionados à Computação nas mais diversas atividades do seu cotidiano.

\section{OBJETIVOS}

Este trabalho tem como objetivo principal investigar qual a contribuição que o ensino da lógica de programação pode acarretar no desenvolvimento escolar dos estudantes de Ensino Médio e qual o impacto que pode ser observado na vida deles diante deste aprendizado, levando-se em conta o uso dos conceitos essenciais da Computação e da lógica de programação aplicados de forma prática na vida dos estudantes. Para isso, a pesquisa visa utilizar os mais diversos tipos de materiais propostos pelos autores de trabalhos relacionados publicados em eventos e revistas de referência, investigando a eficiência dos métodos, os índices escolares em regiões que já passaram pela implementação de iniciativas dentro deste contexto e a opinião dos estudantes, dentre outros fatores. Ao longo do estudo, também pretende-se publicar as sínteses e resultados obtidos com a pesquisa em eventos na área da Informática na Educação, podendo assim tornar estes dados públicos para uso de docentes e demais interessados em analisar os casos de sucesso em projetos semelhantes.

\section{METODOLOGIA}

Para que seja feita a investigação proposta, uma revisão sistemática da literatura apoiada em anais de eventos e periódicos de referência na área de Informática na Educação foi desenvolvida, em busca de iniciativas já realizadas, tendo como público-alvo os alunos do Ensino Médio. As buscas foram feitas:

- $\quad$ Nos periódicos do Simpósio Brasileiro de Informática na Educação (SBIE);

- $\quad$ Na Revista Novas Tecnologias na Educação (RENOTE);

- $\quad$ Nos workshops do Congresso Brasileiro de Informática na Educação (WCBIE);

- $\quad$ Na Revista Brasileira de Informática na Educação (RBIE);

- E na revista Observatório.

Todas as buscas foram realizadas nas edições que compreendem os anos de 2015 e 2019, utilizando os termos pré-estabelecidos, que deveriam estar contidos nos resumos, títulos ou palavras-chave como critério de inclusão (CI), conforme consta na Tabela 1, com os seus respectivos significados, sugerindo que todos eles podem ser utilizados nas salas de aula em diversas áreas do conhecimento, abrangendo diversos conceitos, dentre eles os métodos de resolução de problemas, por exemplo. Em seguida, foi devidamente realizada a leitura de cada um dos artigos, com uma seleção a partir dos critérios de exclusão (CE), que também são apresentados na Tabela 1, restando apenas aquelas publicações que trouxeram relatos de iniciativas educacionais que possuíam como público-alvo os educandos do Ensino Médio.

Visto que grande parte dessas publicações não possui depoimentos dos participantes envolvidos, uma análise de campo também se demonstrou necessária, procurando pela opinião e impacto observado diretamente pelos estudantes nas escolas de Ensino Médio. Para esta análise, foram selecionadas seis perguntas realizadas por meio de um questionário online, pelos estudantes devidamente e formalmente autorizados por seus responsáveis através do preenchimento dos termos de consentimento. Este questionário, respondido de forma totalmente anônima por todos os envolvidos, abrange as perguntas $(\mathrm{P})$, conforme apresentado na Tabela 2.

Após o levantamento dos dados obtidos pelas duas análises (revisão sistemática e entrevistas), os resultados serão apresentados, e a possibilidade de novos estudos é cogitada, com foco maior em fatores que podem se mostrar relevantes, como a região em que são implantadas as iniciativas, o desempenho escolar dos estudantes que responderam os questionários, os tipos de iniciativas relatadas nos artigos, bem como aquelas 
que os estudantes gostam ou possuem interesse, dentre outros aspectos, considerando principalmente a relevância dos conhecimentos essenciais de Computação e lógica de programação para a realização de tarefas básicas nas rotinas diárias de cada participante.

Tabela 1. Critérios de Inclusão e de Exclusão utilizados nesta revisão sistemática. Fonte: autoria própria (2020)

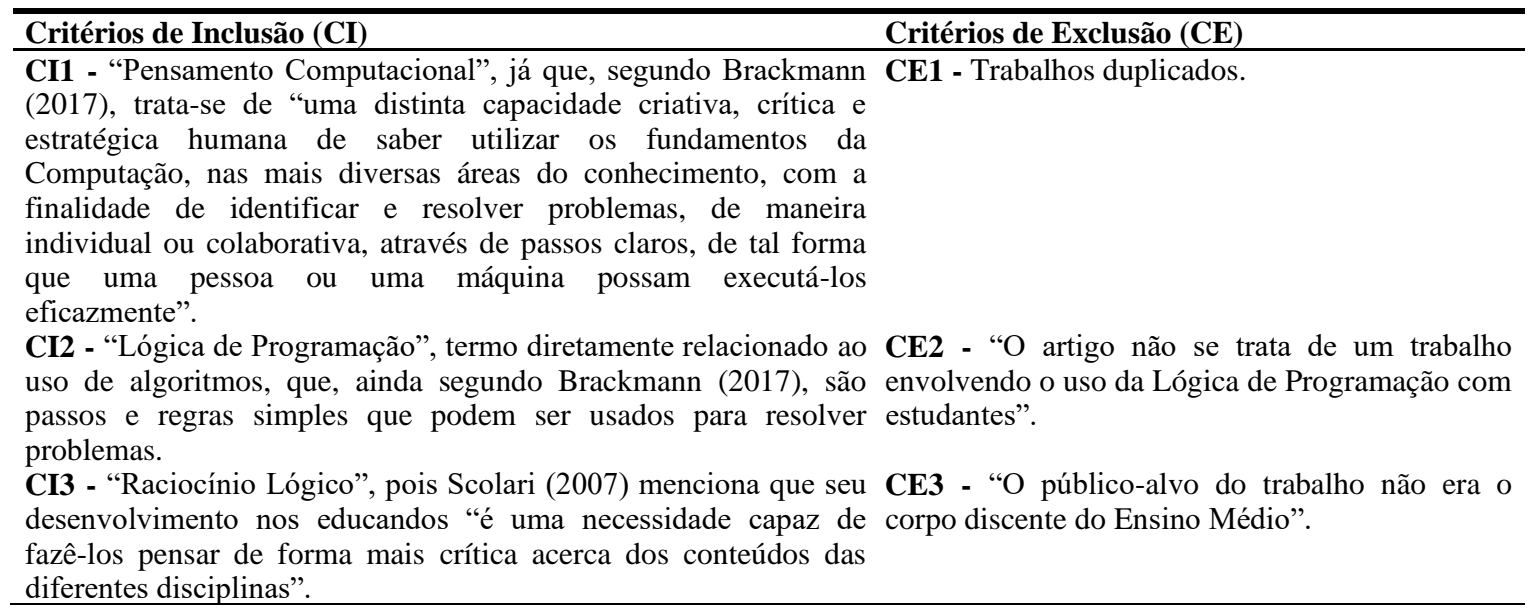

Tabela 2. Perguntas realizadas na pesquisa de campo. Fonte: autoria própria (2020)

\begin{tabular}{ll}
\hline Perguntas & Questão \\
\hline P1 & "Já possuiu algum contato com lógica de programação no Ensino Fundamental? (Sim/Não)" \\
P2 & "Já possuiu algum contato com lógica de Programação no Ensino Médio? (Sim/Não)" \\
P3 & "Se você respondeu sim para a pergunta acima: este contato trouxe algum impacto (em decisões) na sua \\
vida? Qual?" & \\
P4 & "Você possui interesse em ter contato com a lógica de programação? (Sim/Não)" \\
P5 & "Como foi seu desempenho em Língua Portuguesa no último ano em sua vida escolar? \\
& (Péssimo/Ruim/Regular/Bom/Ótimo)" \\
P6 & "Como foi seu desempenho em Matemática no último ano em sua vida escolar? \\
& (Péssimo/Ruim/Regular/Bom/Ótimo)"
\end{tabular}

\section{RESULTADOS}

\subsection{Revisão Sistemática}

$\mathrm{Na}$ Tabela 3 é possível visualizar os artigos que foram encontrados nos 5 veículos de investigação e com o uso dos 3 critérios de inclusão considerados (CI1, CI2 e CI3).

Tabela 3. Artigos encontrados de acordo com os Critérios de Inclusão. Fonte: autoria própria (2020)

\begin{tabular}{llll}
\hline Veículo de comunicação & CI1 & CI2 & CI3 \\
\hline WCBIE & 56 & 29 & 23 \\
SBIE & 35 & 15 & 8 \\
RENOTE & 9 & 9 & 6 \\
RBIE & 6 & 2 & 2 \\
Observatório & 3 & 1 & 0 \\
\hline
\end{tabular}

Aplicando o critério de exclusão CE1, essa tabela resume-se em um total de 265 trabalhos, que novamente foram reduzidos para apenas 41 iniciativas quando aplicados os critérios de exclusão CE2 e CE3, podendo sugerir a confirmação da afirmação apontada por alguns autores em relação à carência de atividades relacionadas à lógica de programação no Ensino Médio, mostrando assim que existe a demanda e a necessidade de iniciativas que contemplem e abordem de forma satisfatória este assunto nas escolas brasileiras, para que os estudantes se sintam melhor preparados para lidar com a tecnologia em suas vidas, independente da área de aplicação. 


\subsubsection{Ausência de Iniciativas}

Em dois periódicos analisados não foi observada a ocorrência de iniciativas que contemplem o objetivo da busca. Na revista Observatório, em todas as suas edições lançadas no período entre 2015 e 2019, foram encontrados apenas 3 trabalhos com os termos pré-definidos, mas nenhum deles estava direcionado a iniciativas envolvendo o uso da lógica de programação para estudantes do Ensino Médio. Com esta análise, observou-se que embora a revista englobasse trabalhos que promoviam a inclusão digital, eles estavam ligados ao uso das novas Tecnologias Digitais da Informação e Comunicação (TDIC), mas sem foco no desenvolvimento das mesmas, podendo ser instigado o questionamento de que alguns autores ainda não veem o Pensamento Computacional como um conceito interdisciplinar, conforme defende Brackmann (2017). Já na Revista Brasileira de Informática na Educação, foram encontrados 8 trabalhos compreendidos no mesmo período, que se tratavam majoritariamente de trabalhos desenvolvendo o Pensamento Computacional com estudantes que já cursavam a graduação ou para o Ensino Fundamental, além de uma revisão sistemática. Ou seja, novamente não foram encontradas iniciativas voltadas para o Ensino Médio na revista mencionada.

Quanto ao tipo de atividade proposta nestes 41 trabalhos selecionados, pode-se dividir em três grandes grupos, conforme a Tabela 4.

Tabela 4. Relação do tipo de iniciativas com estatísticas em seus resultados nos anais dos workshops do CBIE, SBIE e revista Renote. Fonte: autoria própria (2020)

\begin{tabular}{ll}
\hline Iniciativas utilizadas & $\%$ \\
\hline Software lúdico & $41,5 \%(17 \mathrm{de} 41)$ \\
Oficina de programação & $41,5 \%(17 \mathrm{de} 41)$ \\
Oficina de robótica & $17,1 \%(7 \mathrm{de} 41)$ \\
\hline
\end{tabular}

Outro fator que foi de grande relevância para o levantamento de dados foi a regionalidade dos projetos encontrados, observada na Tabela 5, mostrando que a predominância das iniciativas está na região Sul do Brasil, seguida pela região Nordeste, que possui seis trabalhos a menos, enquanto apenas um trabalho foi encontrado, representado por $2,4 \%$, na região Centro-Oeste. Além da pequena quantidade de trabalhos encontrados nessa região, ainda existe a informação de que esse único trabalho foi publicado apenas no ano de 2019, ou seja, não houveram publicações de autores da região Centro-Oeste do Brasil nestes periódicos em edições compreendidas entre 2015 e 2018.

Tabela 5. Relação dos 41 projetos destinados ao Ensino Médio por localidade de aplicação em cada região do Brasil Fonte: autoria própria (2020)

\begin{tabular}{lccccc}
\hline Região & Sul & Sudeste & Norte & Nordeste & Centro-Oeste \\
\hline $\mathrm{N}^{\circ}$ de iniciativas & $18(43,9 \%)$ & $5(12,2 \%)$ & $5(12,2 \%)$ & $12(29,3 \%)$ & $1(2,4 \%)$ \\
\hline
\end{tabular}

Essa relação entre os trabalhos desenvolvidos em cada região do Brasil instigou duas novas análises. A primeira foi com base nos dados do IDEB das regiões com menor e maior número de trabalhos. Foi observada uma relação entre estes dados e o número de projetos envolvidos nas regiões, considerando a existência de uma diferença de 0,6 pontos na expectativa para 2019 entre a região com maior número de iniciativas (Sul), em que são esperados 5 pontos em escolas estaduais no próximo exame, e a região com apenas uma iniciativa (Centro-Oeste), que espera um índice de 4,4 pontos na mesma rede de ensino. Um fato como este sugere a continuidade da pesquisa bibliográfica a fim de descobrir se é válida a relação entre a implementação da lógica de programação nas salas de aula do Ensino Médio, sugerindo um aprofundamento na investigação para identificar se o maior índice do IDEB pode estar diretamente relacionado a um número maior de iniciativas envolvendo a lógica de programação realizadas na região avaliada. Já a segunda análise trata-se de uma relação entre o número de artigos publicados por ano, buscando observar se houve uma diferença significativa entre o número de publicações ao longo do período considerado para a pesquisa, entre os anos de 2015 e 2019, conforme apresentado na Tabela 6. Nessa pesquisa, foi observado que, com exceção da revista RENOTE, que possuiu periódicos publicados nos 5 anos que a pesquisa abrangeu, mas só possui iniciativas que abrange todos os CI e CE nos dois últimos anos e das revistas RBIE e Observatório que em nenhum dos anos pré-estabelecidos foram encontrados artigos para a análise, não há uma constância, nem menos um aumento de iniciativas ao longo dos anos, como também pode-se ver na Tabela 6 . Nos periódicos do SBIE, por exemplo, o ano mais recente pesquisado não contou com nenhum artigo, enquanto já havia sido totalizados 12 trabalhos neste periódico nos anos anteriores. 
Tabela 6. Relação do número de projetos por ano de publicação nos periódicos analisados, após a aplicação dos critérios de inclusão e exclusão. Fonte: autoria própria (2020)

\begin{tabular}{lllllll}
\hline Ano & WCBIE & SBIE & RBIE & RENOTE & Observatório & Total \\
\hline 2015 & 4 & 3 & 0 & 0 & 0 & 7 \\
2016 & 3 & 1 & 0 & 0 & 0 & 4 \\
2017 & 5 & 4 & 0 & 0 & 0 & 9 \\
2018 & 3 & 4 & 0 & 4 & 0 & 11 \\
2019 & 7 & 0 & 0 & 3 & 0 & 10 \\
\hline
\end{tabular}

\subsection{Pesquisa de Campo}

Na pesquisa de campo, 334 respostas foram obtidas com estudantes de uma instituição de ensino que oferta dois cursos técnicos integrados ao Ensino Médio (Informática e Administração). As demais foram obtidas por meio de divulgação via internet, devido ao isolamento social e a inatividade das instituições de ensino para prevenir a propagação do novo Coronavírus. Somando-se todas as respostas obtidas, foram contabilizadas 366 respostas no total, com os seguintes resultados mediante as perguntas propostas:

- $\quad$ P1: 26,5\% mencionaram já ter em algum momento do Ensino Fundamental o contato com a lógica de programação;

- P2: 63,7\% mencionaram já ter em algum momento do Ensino Médio algum contato com a lógica de programação;

- P3: Dentre as respostas, houveram diversos relatos de rotinas mais organizadas, facilidade na escrita e compreensão de textos, impacto na escolha do curso superior que os estudantes pretendem ingressar, facilidade em outras disciplinas do currículo, desenvolvimento de um pensamento lógico e novos pontos de vista em determinadas metodologias, dentre outros depoimentos.

- $\quad$ P4: 74,9\% mencionaram ter interesse em possuir contato com a Lógica de Programação;

- P5: 70,5\% dos estudantes consideram seu desempenho "bom" ou "excelente" em Língua Portuguesa;

- P6: 64\% dos estudantes consideram seu desempenho "bom" ou "excelente" em Matemática.

Após a obtenção destes dados, foi realizada uma busca pelo IDEB da instituição de ensino em que foi possível aplicar $91,25 \%$ dos questionários, com 334 estudantes, por ser a instituição de quantitativo maior obtido. Assim, buscou-se observar se os índices também coincidiam com as respostas obtidas, e foi encontrada a informação de que o desenvolvimento atingido dessa instituição obteve uma média de 5,5 no IDEB, superior de forma considerável da média estadual, que foi 3,7 e da média nacional, onde foi atingida a nota 3,8, e, ainda, superior ao objetivo nacional do mesmo ano, que teve como média esperada o índice de 4,7, evidenciando assim que o aprendizado de conteúdos relacionados com a lógica de programação pode estar relacionado com um melhor desenvolvimento dos estudantes, diante dos índices apresentados pelo IDEB, privilegiando aqueles que possuem contato com a lógica de programação e tecnologia em sala de aula para a execução de tarefas que exigem um conhecimento mínimo em raciocínio lógico e Pensamento Computacional.

\subsection{Publicações}

Esta seção tem por objetivo incorporar no artigo o histórico da pesquisa para estes resultados e também compartilhar com leitores e demais interessados (professores, estudantes, etc.) de locais públicos onde podem ser encontrados os resultados para análise de dados e a obtenção de informações pertinente para a elaboração de projetos semelhantes.

Cumprindo o objetivo de publicar os dados desta pesquisa em eventos, para que eles possam ser utilizados por pessoas interessadas em pesquisar na área, cada etapa deste estudo já participou de eventos e feiras nacionais e internacionais, conforme a Tabela 7. 


\section{CONCLUSÃO}

Ao longo da pesquisa, os objetivos propostos estão sendo atingidos com êxito. No entanto, pretende-se que o estudo seja ampliado, com o intuito de coletar mais dados para proporcionar uma maior precisão nos resultados, em busca de mais plataformas de pesquisa que possam conter trabalhos sobre o uso da lógica de programação para o Ensino Médio. Além disso, parte desta coleta foi prejudicada devido ao não funcionamento das escolas de Ensino Médio como método de prevenção da propagação do novo Coronavírus, impossibilitando a realização dos questionários em outras instituições de forma integral, conforme proposto inicialmente, a fim de ampliar os resultados deste trabalho.

Tabela 7. Eventos e periódicos em que a pesquisa foi publicada. Fonte: autoria própria (2020)

\begin{tabular}{lll}
\hline Evento & Data da publicação & Local \\
\hline XIX Congreso Internacional de Investigación Educativa & Junho/2019 & Madrid/Espanha \\
VII IFCITEC (premiação de 3 $3^{\circ}$ lugar) & Setembro/2019 & Canoas/RS/Brasil \\
V Workshop de Ensino em Pensamento Computacional, Algoritmos e e Novembro/2019 & Brasília/DF/Brasil \\
Programação do Congresso Brasileiro de Informática na Educação & & \\
XXIV Congreso Internacional de Informática Educativa & Novembro/2019 & Arequipa/Peru \\
Revista Scientia Prima da Associação Brasileira de Incentivo à Ciência & Maio/2019 & Não se aplica \\
I Feira Brasileira de Jovens Cientistas (premiação de 3 $3^{\circ}$ lugar) & Junho/2019 & Virtual \\
$7^{\circ}$ Encontro Nacional de Computação dos Institutos Federais & Aguarda publicação & Virtual \\
\hline
\end{tabular}

Sendo assim, pretende-se então ampliar a revisão sistemática deste trabalho, principalmente enquanto não é possível dar continuidade à pesquisa de campo, investigando outros veículos de comunicação amplamente utilizados na literatura. Assim, será possível abranger outros periódicos de outras revistas e eventos, buscando complementar os resultados já obtidos e encontrar iniciativas em diferentes tipos de comunidades no Brasil, além da possibilidade de buscar por eventos internacionais, a fim de comparar dados de outros países com os já encontrados.

Uma nova possibilidade de tentativa para abranger diferentes pontos de vista e aprimorar os dados apresentados na análise está sendo cogitada. Nela, existe a intenção de também analisar eventos internacionais para a revisão sistemática, contribuindo com dados de diferentes países, realizar entrevistas diretas com educandos e educadores que possuíram contato com lógica de programação, a fim de entender em quais aspectos este contato pode agregar em suas formações, além de investigar métodos pedagógicos de inclusão de estudantes com limitações intelectuais, visando apresentar alternativas não apenas para desenvolver o sistema educacional, mas também para incluir um maior número de educandos contemplados, observando assim se de fato o contato com a lógica de programação pode contribuir para as tarefas do cotidiano na vida dos estudantes que foram contemplados com a abordagem deste conteúdo no Ensino Médio.

\section{AGRADECIMENTOS}

Agradecemos ao Instituto Federal de Educação, Ciência e Tecnologia do Rio Grande do Sul que por meio do Edital IFRS no 71/2019 financiou a publicação do artigo e a participação da autora principal no evento. Agradecemos também ao Conselho Nacional de Desenvolvimento Científico e Tecnológico (CNPq) que financiou a realização dessa pesquisa por meio do edital IFRS nº 24/2019. 


\section{REFERÊNCIAS}

Brackmann, C. P. e Barone, D. A. C., 2017. "Desenvolvimento do pensamento computacional através de atividades desplugadas na educação básica". Tese (Doutorado). Universidade Federal do Rio Grande do Sul. Porto Alegre, Brasil, 226p.

Brasscom (2020). "Sobram vagas em tecnologia - crescimento do setor acelera". Disponível em: https://brasscom.org.br/sobram-vagas-em-tecnologia-crescimento-do-setor-acelera (Acesso realizado em 22 julho 2020).

Carvalho, J., Netto, J. F., Almeida, T., 2017. "Revisão sistemática de literatura sobre pensamento computacional por meio de objetos de aprendizagem.". Anais do XXVIII Simpósio Brasileiro de Informática na Educação. Brasília, Brasil, p. 223-232.

Costas, R. (2015). "Modelo de escola atual parou no século 19, diz Viviane Senna". Disponível em https://www.bbc.com/portuguese/noticias/2015/06/150525_viviane_senna_ru (Acesso realizado em 23 julho 2020).

Gomes, T.; Melo, J., 2013. "O Pensamento Computacional no Ensino Médio: Uma Abordagem Blended Learning”. Anais do XXI Workshop sobre Educação em Computação. Maceió, Brasil.

Heintz, F., Manilla, L., Färnqvist, T., 2016. "A Review of Models for Introducing Computational Thinking, Computer Science and Computing in K-12 Education”. Anais da IEEE Frontiers in Education Conference (FIE). Erie, EUA.

IDEB (2020). "IDEB - Resultados e metas". Disponível em: http://ideb.inep.gov.br/resultado/ (Acesso realizado em 22 julho 2020).

IMD (2020). "IMD World Competitiveness Center". Disponível em: https://www.imd.org/wcc/world-competitivenesscenter-rankings/world-competitiveness-ranking-2020/ (Acesso realizado em 22 julho 2020).

Idoeta, P. A. (2019). "Viviane Senna: Brasil ainda não fez lição de casa do século 19 na educação". Disponível em https://www.bbc.com/portuguese/brasil-48631414 (Acesso realizado em 23 julho 2020).

Lyceum (2019). "Pisa - Ranking de educação mundial: entenda os dados do Brasil". Disponível em: https://blog.lyceum.com.br/ranking-de-educacao-mundial-posicao-do-brasil/ (Acesso realizado em 22 julho 2020).

Sales, C. V., Vasconcelos, M. A. D. M., 2019. "Ensino Médio Integrado e Juventudes: desafios e projetos de futuro". Revista Educação \& Realidade. V. 41, nº 1, p. 69-90. Porto Alegre, Brasil.

Schuhmacher, E. Rompelato, D., Schuhmacher, V., 2016. "O Desenvolvimento do Pensamento Computacional no Ensino Médio por meio de Ambientes de Programação". Anais da XIV International Conference on Engineering and Technology Education. Salvador, Brasil. p 234-243.

Scolari, A.T., Bernardi, G., Zanki, A., 2007. "O Desenvolvimento do Raciocinio Lógico Através de Objetos de Aprendizagem”. Renote - Revista Novas Tecnologias na Educação, Porto Alegre, Brasil.

Silva, H. B. P., 2011. "Análise comparada da estrutura curricular do curso de pedagogia em duas instituições: Universidade de Brasília e Instituto Nacional de Educação de Singapura". Monografia (Licenciatura em pedagogia). Universidade de Brasília. Brasília, Brasil, 101 p.

Palfrey, J., Gasser, U., 2011. "Nascidos na era digital: entendendo a primeira geração de nativos digitais”. Artmed. Porto Alegre, Brasil.

Rodrigues, S., Aranha, E., Silva, T. R, 2018. "Computação desplugada no ensino de programação: Uma revisão sistemática da literatura." Anais do XXIX Simpósio Brasileiro de Informática na Educação. Fortaleza, Brasil. p. 417-426. 